\title{
Optimal decisionmaking or optimal trouble-shooting?
}

\author{
G.J. Hofstede \& A.J.M. Beulens \\ Wageningen Agricultural University, the Netherlands \\ Dept. of Computer Science \\ e-mail hofstede@rcl.wau.n! \\ fax $(+31) 8370.84731$
}

\begin{abstract}
The aim of this paper is to view the notion of optimal decision support from the practical perspective of a user - manager. Using this perspective forces one to conclude that optimization techniques in the classical OR sense do not necessarily lead to improved, let alone optimal, decision support. The organizational and environmental contingencies determine what kind of DSS, if any, are feasible to support decisionmaking in a given situation.

For the area of tactical / operational planning decisions, we propose a simple assessment tool to judge which type of DSS could be appropriate. The tool investigates eight aspects from both the context and the object system in a qualitative manner. The possibilities of the tool are demonstrated for two cases: cultivation planning in potted plant nurseries, and physical distribution in a large production company. The present status of the tool is tentative. The tool predicts that in most planning situations highly interactive planning systems, in which the planner has primacy over the automated system, are called for.
\end{abstract}

\section{Introduction}

This Conference bears the title "Optimization-based Computer-Aided Modelling and Design". The present paper deals with the notion of optimization in the context of Decision Support Systems. It is written from the point of view of a manager using a DSS for a real-world planning problem situation. The types of problem situations considered are tactical or operational: repeatedly occurring problems with a marked combinatorial aspect. What does optimality mean for the user, that is, the planner or manager? Is it attainable, and what does it consist of? For instance, optimality from the user's viewpoint requires at the very least that the decision reached with help of the DSS can actually be implemented, and that it fits with related decisions. In our experience organizational decisionmaking is usually not a neatly partitioned activity. On the contrary, it is more like a whirlpool: no decision can be singled out, and it is very hard to grasp how and why decisions are arrived at. More often than not, ad hoc troubleshooting is what planners do, rather than optimizing isolated decision-making processes. Our experience with the cases of production planning in potted plant nurseries and of physical 
distribution in a large organization is that optimality in an organizational context is something quite different from the mathematical notion of optimality familiar to Operations Researchers. If DSS designers intend to strive for optimal decision support they have to be aware of the environmental and organizational context in which their systems will be used. The result may well be a system which is childishly simple from a designer's point of view, but which fits into the organization and its decision processes, and satisfies performance requirements, rather than a sophisticated optimizing system which is not accepted and cannot be integrated into existing decision making practices.

In the next sections we shall elaborate on our views about organizational decisionmaking and DSS, concentrating on tactical / operational planning decisions. We shall mention some DSS literature in support of our view that the context of a planning situation is of paramount importance when designing a DSS. Then we present the tentative tool for assessing DSS feasibility in a given situation. We illustrate the use of the tool for potted plant production planning and for physical distribution in a large company. Finally a discussion about the merits of the tool and directions for further research concludes the paper.

\section{Optimal organizational decisionmaking}

\section{Prescriptive and descriptive}

Theories of organizational decisionmaking come in two orientations: prescriptive and descriptive. Either implicitly or explicitly all such theories contain a mix of these two orientations.

The prescriptive or normative theories stress that partitioning activities and predefining a goal structure should act as a basis for decisionmaking, and such theories even define procedures, models and algorithms to be applied for decisionmaking (e.g. Anthony 1965, Kampfraath \& Marcelis 1981, Lindley 1985).

Descriptive approaches stress the mess which is present in organizations: decisions interact, work is fragmented, goals are never made explicit and are of a qualitative and/or quantitative nature, or they are invented after the fact to justify decisions. In other words, the conditions under which formal methods such as mathematical optimization are of value may not be met, at least when considering organizational decisionmaking as a whole (e.g. Cyert \& March 1963, Lindblom 1959, March \& Olsen 1976, Mintzberg 1973).

In accordance with the descriptive approaches we define organizational decisionmaking as a complex of interrelated activities in which decisions do not stand by themselves. As such many decisions defy attempts at exhaustive formal modelling, for instance in a DSS. However, structuring these activities in the sense of the prescriptive theories is generally desirable and DSS can play a role in this. We define a decision as the outcome of a decision problem. A decision problem is a conceptual entity abstracted from the organizational processes. A decision problem has goals and variables and operates on an object system (OS). To indicate a decision problem which is not yet clearly conceptualized, together with its organizational context we use the term decision situation. 
DSS and unstructuredness

Many authors of DSS papers, see van Dissel et al.(1990), seem to agree on a few key characteristics of DSS. DSS are supposed to support decision makers, rather than replace them. Further DSS are supposed to be concerned with support to solve semi- and unstructured problems. Unstructuredness of the problem may be defined as the degree of incompleteness of the knowledge of the decisionmaker of one or more of the following:

- the demarcation of the object system from its relevant environment, as well as the demarcation between relevant and irrelevant environment.

- the initial state of the object system, including available resources,

- the goals to be attained and the attributes of relevance attached to these goals,

- decision alternatives and their expected consequences as generated by a (set of) model(s) of the OS.

- procedures and methods to obtain necessary information, to specify goal attributes and to generate and evaluate decision alternatives using available MIS and DSS.

This enumeration shows that unstructuredness can reside in various aspects of a decision problem. In fact, since a decision problem is a conceptualization, by definition unstructuredness resides in the mind of the conceptualizer. If a problem owner and a DSS designer both contemplate a problem situation they may arrive at different conceptualizations. As a problem owner learns about his problem the problem becomes more structured, and this is entirely a conceptual learning process which does not affect the problem situation as it would be perceived by a third person. It follows that a good DSS is most likely amongst others an 'LSS', or learning support system, for the problem owner. By analogy the design process of a DSS is also a learning process, gone through by designer and problem owner, which hopefully results in a shared conceptualization.

\section{DSS and improved decisionmaking}

DSS are intended to improve decisionmaking. This means that, to begin with, they must be used. It is not realistic to expect organizational decisionmaking, which is rooted in human social behaviour and cognitive limitations, to change overnight in order to accommodate a DSS. Rather a DSS must fit into the existing pattern or demand modest changes, depending on the will to adopt it (see e.g. Beulens \& Hofstede 1990). It is then that organizational decisionmaking can be 'optimized', or rather, improved.

Frequently there are very low-brow practical impediments to improved decisionmaking. Beulens (1990) sums up a number of these impediments. Basically they come down to the fact that in many cases, practical functional and performance requirements for DSS have not been properly defined. Such requirements are the yardstick for measuring quality, or if you will optimality, of the DSS. They can be derived from aspects such as the organizational and environmental context, the tasks and relative priority of these of the user, the way in which these tasks are being performed and interact (fragmented decision making processes), the problem and systems knowledge of the user, the information sytems that are to be interfaced with. The decision itself as a modelled abstraction from the organizational whirlpool plays no more than a modest role. 


\section{Organizational contingencies}

The nature of an organization's decisionmaking processes sets conditions for DSS development and DSS use. The people, procedures, and production processes in an organization, its culture and its environment all play a role. If an organization strongly adheres to prescriptive management theory, e.g. decisions are explicitly defined, procedures to solve them are explicit, high-quality data are collected, and the like, then optimization in the mathematical sense may be feasible. If, on the other hand, decisionmaking is informal, fragmented, lacking accurate data, then mathematical optimization is most definitely not feasible. Most cases will be intermediate and ask for interactive, dynamically robust DSS.

Reports from practitioners who have developed DSS frequently make mention of organizational factors which have impeded use of the system, or of downward adjustment of the sophistication of a system during the implementation phase (e.g. Institution of Electrical Engineers 1990). In many cases this can be attributed to incorrect or incomplete assessments of the kinds of functional and performance requirement previously indicated. Users, on the other hand, are typically content when at least clerical tasks are taken off their hands by a DSS but are often distrustful of the outcome of model-based reasoning or computations generated by a DSS. They are certainly not content if (clerical) tasks are to be performed when using a system when no real benefit is perceived.

\section{Example of school timetabling}

For example, take the case of Verbraeck (1990). This author has developed and successfully marketed a lesson-planning system for large schools. His experience when working with planners in schools made him aware of the misfit which can occur between a human planner and an automated DSS. On p. 209 Verbraeck remarks:

"Manual lesson-planning has a number of advantages over automated planning. The planner has a clear understanding of the planning situation. Difficulties can be tackled in parallel. The planner knows where the initial information can be altered without creating trouble. The planner is able to identify partial problems, blocking lessons, and small puzzles that can be solved apart. The time it takes to make a schedule can be controlled by the planner. The criteria the schedule has to meet can be changed when the process threatens to take longer than expected."

The keywords behind this enumeration seem to be flexibility and problem expertise. However manual planning also has severe drawbacks such as inconsistencies and errors, excessive time consumption. In his planning system Verbraeck has attained the benefits of automation without losing too much flexibility by designing an algorithm with the following properties (p.209):

- The planner is able to edit the schedule manually. The algorithm does not affect manually created plan parts.

- The search time is limited and under control of the planner.

- In the allotted time a schedule is always presented whether all lessons are included or not.

- With growing search time, the quality of the schedule increases.

- The algorithm works even if there are inconsistencies in the input data.

Summarizing we can say that Verbraeck's system is very much a satisficing rather than an optimizing system, and that it leaves the planner in full control of his planning strategy. These are obviously important assets in an environment which is as unpredictable as a school and in which constraints are so manifold but hard to quantify. 
A strong viewpoint about the misfit between many automated planning systems and actual planning practice in organizations is expressed by McKay et al. (1989). These authors carried out an investigation among over 300 practitioners of job-shob scheduling and found that not one of the planning situations in which these persons worked contained any of the following properties:

- well-known and stable manufacturing process

- $\quad$ simple goals not affected by hidden agendas

- $\quad$ predictable and reliable setup and processing times

- relatively short cycle times to allow most work to start and complete without interruptions

- accurate and complete drawings, routings, and bills of materials

- reliable, stable, and accurate product demand forecast

- known material quality, quantity, and arrival times.

Obviously these 300 planning situations are ill-suited to formal modelling and full automation. McKay et al. remark (p.172):

"In general, the world of scheduling remains as it was in the fifties and sixties. Neither Operations Research nor Artificial Intelligence has made a significant impact on how scheduling is done in the real world."

Any conference on DSS, OR or AI can serve to illustrate this remark. For instance at the first specialized IFORS Conference on DSS held in March 1991, it was remarked in keynote speeches that $O R$ had drifted from solving real problems to finding solutions looking for problems. Recently, the concept of DSS has provoked a renewed interest in OR. Getting or keeping the OR research community in touch with organizational needs was perceived as a major challenge. At present the situation is not ideal; as J. Sviokla mentioned in a panel session the ratio between research prototype systems to beta-test versions to organizationally implemented systems is about 100 to 10 to 1 .

\section{An assessment tool for DSS in planning}

It is our conviction that a large majority of tactical / operational planning situations of very diverse nature will remain intractable for models without assistance by human planners. At the same time, given flexible, incremental models and an awareness of the importance of organizational contingencies, much improvement should be possible.

We have attempted to operationalize the above description of organizational decisionmaking, generating a tentative tool which can be used during a quick scan to assess chances for DSS development in a given decision situation. In fact the tool was born out of frustration of one of us who unsuccessfully tried to get potted plant growers to accept normative support. The tool investigates contingency factors that have to be taken into account when specifying functional and performance requirements for DSS. Given a decision area of concern in the area of tactical and operational planning we propose to rate eight aspects of the problem context. Aspects that concern the organization, the environment of the organization, and the direct decision making 
context are grouped in the category "context characteristics", aspects that are related directly to the object system to be controlled are grouped in the category "object system characteristics".

\section{Context characteristics}

1 How large is the organization?

In a very small organization the tactical level is not separated from the strategic and operational levels, because the same person or group of persons attend to all levels. This means strong 'contagion' of the decision by almost all other decisions. Plans tend to be informal or even absent. If on the other hand the organization is large then the planning process becomes more detached from other organizational decisions and there may exist formal organizational planning procedures.

2 Is there a specialized planner?

In the absence of a specialized planner, planning is often done by someone with many other obligations. It may be done in a quick-and-dirty, ad hoc way, especially if the other responsabilities of the person who does the planning are perceived as more important or more fun. If there is a person or group of persons specifically concerned with the planning situation - which often occurs in larger organizations then usually the planner is an expert, spends much time on planning, likes to do a good planning job, and has an interest in automated support (but perhaps also fear to be chased from his job by a computer).

3 How dynamic is the environment?

In a sector where the environment (in a broad sense) is dynamic and unpredictible, decisionmaking has to be highly responsive to external signals. If the environment is comparatively stable, there is more to gain by an orientation towards improving the efficiency of internal operations. Often an organization is to some extent free in defining its environment, as in the case of a potted plant grower choosing to sell through private channels or via the auction.

4 What is the frequency of the decisionmaking process?

Tactical and operational planning decisions they are by definition recurrent. But the point is still relevant if formulated differently: how often does the decision making process occur before a decision situation changes so much that it must be considered to be different from the current decision problem? In most organizations changes are fairly frequent. For instance, if a planning decision is taken yearly and major organizational changes occur once every three years, then developing a DSS to support the generation of these plans may not be cost-effective.

\section{Object system characteristics}

5 How predictable is the object system?

The better an OS can be predicted, the better an automated model of the OS can function as a means to generate decision alternatives and expected consequences thereof. An OS can be considered predictable when a planner accepts a model of the OS and its forecasts within a specified context. In fact when the OS is not predictable this can mean one of two things: either it is known that there are limits 
to predictability, as with the weather influencing crop growth, or the complexity is such that there currently is no predictive model but it remains open whether a predictive model will be found. Although in both cases a simulation model can be of great value, the potential predictive benefits of a model are larger in the latter situation.

6 Are people concerned?

This point is no more than a special case of the previous one but so important that it deserves special mention. As soon as people's interests are affected by a plan then a variety of constraints come into play. Both during plan generation and during plan execution there will be soft constraints related to persons. Especially if planner and planned have some relationship then interpersonal considerations will play an important part.

7 How controllable is the object system?

To some extent, adaptive control measures can counteract unpredictability, thus making planning feasible. An important assumption to make model-based DSSs feasible is that planners using the DSS are able to generate plans that can actually be realized. For instance, in potted plant culture one can counteract unfavourable radiation circumstances by artificial lighting.

8 Are reliable data available?

However predictable and controllable a process, if data needed by a model are not available, not reliable, or too expensive, then the output of a DSS based on this model is of limited value. This point directly relates to the availability and accessibility of information primarily contained in operational information systems (OIS). Besides it is important to mention here that there may exist an actuality and relevancy gap between the DSS and the systems to be interfaced with. By actuality gap we refer to the fact that in many cases OIS are up to date to for instance TO, the current time is $\mathrm{Tc}$ and the DSS requires data up to $\mathrm{T} 1$ with $\mathrm{T} 1>\mathrm{Tc}>\mathrm{TO}$. With relevancy gap we refer to incompatibilities that may exist between the data models of OIS and DSS.

Operationalizing the scales of the tool

The assesment tool can be applied by scoring each of the eight characteristics on an ordinal scale from 1 to 5 . The following table shows what real-world properties may be associated with the extreme scale values. 


\section{feature}

1 org. size

2 planner

3 environment situation with rating 1

1 to 5 people. At most one allround boss.

Boss does the planning among many other tasks.

Not all relevant variables are known. There are major changes in external conditions on each repetition of the decision making process.

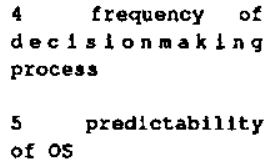

Un1que dectsion

No accepted model of the os and/or essentlal variables are unpredictable within 503.

People are belng scheduled. Their wishes are very important.

Process not controllable.

Data are of unknown quality, often unquantified rumours, hunches, expectations. stuation with rating 5

Over 100 people, Formally defined, clear function separation

Speclalized planner or team; does nothing else.

All varlables are accepted to be known. The conceptualization can cope with the changes of external conditions tmarkets, prices, laws, ....).

Decision process repeated unchanged many times for subsequent planning perlods

Accepted model of OS and all variables are assumed sufficientiy predictable withln allowed t.

No Interests of people are assumed to be affected by the plan in any way.

Process controllable.

Data are quantitative, accurate, complete, up to date, rellabie.

Table 1: operationalized assessment tool for DSS feasibility

For the time being this assessment tool is in need of empirical validation. A specific point is that some of the elements are probably highly correlated and should therefore be joined. Another point is that some characteristics may be of more importance than others. However, our conviction is that by using the tool critically it can evolve into a valuable and empirically sound instrument for assessing DSS chances. The following examples show how the tool can be applied.

\section{The case of a potted plant nursery}

The first case is production planning in potted plant culture under glass, which has some special properties: small firms with poorly partitioned decisionmaking, unpredictable weather entailing an unpredictable primary process, and unpredictable markets, a dynamic environment all work together to create conditions unfavourable for optimization-based decision support. Because glasshouse space is expensive high space occupation is important. Potted plants typically need to be spaced several times during cultivation, and transport is also expensive. So, to simplify 
somewhat, there is a conflict between space occupation and labour cost, added to the uncertainties mentioned above. A more elaborate description of this planning problem can be found in Hofstede (1990).

We have tried an approach based on user-manipulatable heuristics, and found that even that was too ambitious. An evolutionary approach to DSS, starting with low-brow support, working closely together with users, turned out to be succesful in this case.

As an instance we take the case of a modem, innovative potted plant nursery in the Dutch Westland region, grower R. Grower R. wishes to go quite far in automated support of production planning; in fact they have been experimenting with Linear Programming-based DSS.

The scores for grower $R$ are as follows:

1 Organization: 50 persons, in the process of specializing. Entire management meets weekly. Score 2, going towards 3 .

2 Planning: would-be specialized planner, much needed for other work. Score 3.

3 Environment: Highly dynamic. Markets, technology, products subject to yearly changes, i.e. conditions differ each planning cycle. Score 1.

4

Frequency: Each year has a different greenhouse asssortment, objectives. Yet principle constant over time. Score 2.

5 Predictability: Growth unpredictable due to weather, quality of basic material. Fluctuations up to some $25 \%$ of cultivation time. Furthermore, unexpected events or opportunities may arise. Score 2.

People: Planning has some, but not severe, consequences for labour, especially at peaks. Relation between management and labourers is valuable, so labour conditions are important. Score 4.

Controllability: growth conditions can be controlled by extra lighting, heating, chemical treatment and other means, but costs are considerable. Also, new cultivars tend to show unpredictable reactions to treatments, e.g. ugly flowers in some Spathiphyllum varieties. Practically speaking the process is hard to control and only within tight constraints. Score 2.

Data quality: Registration of production data is automated and suficiently detailed, namely to the individual production batch. But the automated system does not generate the desired reports. Price data are not specific enough, e.g. differences between growers can be significant but are not found in the statistics coming from the auction. Score 2.

There are many low scores of 2 . If the innovations desired by $\mathbf{R}$, are realized, some scores can rise a few points, but not drastically. On this basis we predict that the planner must be very cautious about automating cultivation planning. Optimization should be used with care, because its preconditions do not hold. There are too many factors which are unpredictable, or unknown, or unquantified, or have to be decided ad hoc. A modest approach, aimed at generating feasible and robust plans, possibly at what if-exploration of given plans, seems the highest level of support which fits. Coupling between a planning DSS and actual plan realization data is essential because it saves a lot of clerical work. Currently the only automated support system used in production planning by grower $R$ is a spreadsheet application built by the planner himself. By the way, most of the characteristics of grower $R$ are fairly generic to potted plant nurseries. A smaller or less innovative nursery than $R$ would have had even a much lower 
rating. At a nursery similar to $R$. we implemented a simple planning system which basically consists of a Gantt-chart-like interface with interactive editing facilities.

\section{The case of physical distribution}

The second case is of a large manufacturing firm, with some planning situations for which data accepted to be controllable and high-quality are available. The problem area is physical distribution. One operational physical distribution planning problem is to effectively allocate and dispatch finished products to client-warehouses throughout Europe on a daily basis. The stocks available at the factory warehouses have to be allocated to client-warehouses. Subsequently the allocated products must be assigned to trucks under stacking, volume, and weight constraints. The allocation is based on up-to-date sales forecasts and stock data and aims at equal expected service levels per product over all depots. Over time the logistics managers developed a good insight in the way in which this problem can be dealt with. This does not mean that they developed a perfect optimizing model. It does mean they developed a set of models and solution procedures that are accepted to adequately represent the main quantitative aspects of the problem. It also means that organizational procedures have been developed for parties (factories, country sales-units and warehouses) concerned, which are being accepted and adhered to, for the accurate and timely administration of sales forecasts, actual sales and stock data. Over time for this type of problem a sequence of DSS have been developed and implemented. Versions of these DSS have evolved over time such that the task distribution over planner/user and system has dramatically changed, the level of integration with other physical distribution systems has changed through the use of a shared database, the roles of the planners have changed, etc. In short we may say that over time the scope of functions provided by successive systems increased, and that the way in which specific tasks are being performed have evolved into more sophisticated functions. The sophistication resides in the fact that models, used for the allocation of finished goods to client warehouses and the optimal loading and stacking of these goods into trucks, can generate practical feasible truck-loads that are frequently accepted and dispatched without further planner intervention. The last version of the DSS has been effectively used during a number of years to generate decision scenarios which the users/planners could further refine at a qualitative level. The system has provided the users with structured working knowledge of optimization models and their restrictions. They accept the models and their results as valid in the problem situation and claim that the effective execution of their tasks requires the use of the system. If we look at the context and object system characteristics of the problem situation just depicted it is easy to see that the score for all eight characteristics is high (4 to 5). Within tight organizational constraints the problems and solution procedures have been made structured and are accepted by the planners to be so. Thus there has been a joint process of organizational change and of learning, thanks to which a sophisticated DSS offering model-based support with little planner intervention has been developed and implemented and is being used. 


\section{Discussion}

\section{The assessment tool and DSS interactivity}

The evidence from these two cases suggests that the assessment tool does have a relation with DSS chances and especially with the opportunities for model-based DSS.

At the cost of some oversimplification, the eight characteristics could be projected onto a single support dimension. This dimension concerns the interactivity of a DSS. Interactivity can compensate deficiencies in an automated model, inasmuch as it allows the user to bring in ad hoc elements during a planning session (see Hofstede 1991).Note that our definition of interactivity is stronger than is often the case in papers by $\mathrm{OR}$ practitioners. We define full interactivity as the condition in which the DSS user can both monitor and overrule the activities of the automated system during the entire planning session. In other words, if the system contains one or more models these models themselves must be interactive. A planning session is defined as the period(s) from entering the planning system till leaving it, associated with one organizational planning cycle and with the generation of one or more distinct plans for the problem instance at hand. No interactivity exists when the user's role is limited to manipulating the input and output of the planning session. In the potted plants case, full interactivity with no normative support was the only acceptable situation for the user. In the physical distribution case, a fully automated model with no interactivity was accepted but only after a learning process and a series of incremental additions to the DSS. We believe that some growth to normative support can also be expected in the potted plant case.

\section{Limitations of the tool}

A caveat about this assessment tool is appropriate. The tool tells us something about the feasibility of a DSS for a given case, about contingency factors to be taken into account when specifying functional and performance requirements for a DSS, about the type of DSS that could be appropriate, but not about the actual opportunities for getting a DSS built and used! For example, it does not take into account preconditions for DSS success such as innovativity of the organization, or personal acquaintance of the DSS designer within the organization, or the attitude of those who have to pay for the development.

\section{Possibilities of the tool}

By applying the tool to the two cases we illustrated its usefulness. We believe that given more empirical validation it can grow into a valuable aid for a priori assessing chances of DSS development.

A different possible use of the tool is to diagnosticize a given decision situation not with a view to build a DSS but rather to alter the situation itself, e.g. by taking measures to improve the score on one of the aspects.

Whatever the objective of using the tool, the insight to be gained by user and designer of a prospective DSS by considering the planning situation in the light of the tool is valuable. It may be a help in starting the learning process mentioned earlier through which user and designer must go together. 


\section{Final words}

Our experiences with design and implementation of DSS as well as our viewpoint on organizational decisionmaking in general lead us to make a plea for interactive, dynamically robust DSSs for most planning situations. Such DSS will allow the user to improve decisionmaking even in situations that do not allow the use of formal models. They allow incremental development of more sophisticated DSS in situations that do allow the use of formal models. The assessment tool presented in this paper can give an indication which type of models and interfaces are appropriate for a given planning situation.

We hope that the first effect of the tool proposed here is to generate a fruitful exchange of ideas among DSS researchers and practitioners. Perhaps the perspective on decision support to which the paper's title refers is even more important than the assessment tool itself.

\section{References}

Anthony R.N. (1965)

Planning and Control Systems, a Framework for Analysis, Harvard University, Boston.

Beulens A.J.M. (1990)

More powerful and user-friendly DSS by incorporating ES technology? in: D. Ehrenberg, H. Krullmann \& B. Rieger (eds), Wissensbasierte Systeme in der Betriebswirtschaft, Ehrig Schmidt Verlag, Berlin.

Beulens A.J.M. en Hofstede (1990)

Information Systems and DSS in Agriculture: Current status and future possibilities, in: W.H.G.J. Hennen et al. (eds), Informatica-toepassingen in de Agrarische Sector, voordrachten VIAS-Symposium 1990, Agro-Informatica-reeks no 4, pp. 87-94 (in Dutch, English abstract).

Cyert R.M. \& J.G. March (1963)

A behavioral theory of the firm, Prentice-Hall, Englewood Cliffs, New Jersey.

Dissel H.G. van, H.P. Borgman \& A.J.M. Beulens (1990)

Task-Allocation between DSS and Problem Owner: The example of Box \& Jenkins Time Series Analysis, Decision Support Systems 6, pp 339-345.

Hofstede G.J. (1990)

Interactive Planning System design, a model and an application, Proc. first Int. Conf. on Expert Planning Systems, IEE Conference Publ. 322, the Institution of Electrical Engineers, London, pp. 175-180.

Hofstede G.J. (1991)

Interactive Heuristics, paper presented at the first IFORS-SPC on DSS, Bruges, March 2629, submitted for publication.

Institution of Electrical Engineers (1990)

Proc. 1st IEE Conf. on Expert Planning Systems, IEE Publ. 322, the Institution of Electrical Engineers, London.

Kampfraath A.A. \& W.J. Marcelis (1981)

Besturen en organiseren, Kluwer, Deventer.

Lindblom C. E. (1959)

The Science of Muddling Through, Public Administration Review, 19, 79-88. 
Lindley D.V. (1985)

Making Decisions, 2nd ed., Wiley, London.

March J.G. \& J.P. Olsen (1976)

Ambiguity and Choice in Organizations, Universitetsforlaget, Bergen, Norway.

McKay K., J.A. Buzacott, F.R. Safayeni (1989)

The scheduler's knowledge of uncertainty: the missing link, in J. Browne (ed.), Knowledge Based Production Management Systems, Elsevier Amsterdam.

Mintzberg H. (1973)

The Nature of Managerial Work, Harper \& Row, New York.

Verbraeck A. (1990)

A Decision Support System for timetable construction: automated lesson planning using a special timetabling algorithm, Proc. first Int. Conf. on Expert Planning Systems, IEE Conference Publ. 322, the Institution of Electrical Engineers, London, pp. 207-211. 